\title{
¿Estamos ante un "giro poscompetitivo" en la política de ciencia, tecnología e innovación?
}

FEDERICO UASEN"

\section{Resumen}

Las políticas de ciencia, tecnología e innovación (CTI) suelen poner al crecimiento económico y la competitividad como uno de sus objetivos centrales. En este artículo, planteo que en los últimos años han surgido marcos conceptuales en las políticas de CTI que discuten la adecuación de las políticas convencionales para casos en los que se plantean desafíos sociales específicos. Estos nuevos marcos dan mayor relevancia a los objetivos sociales antes que a las metas económicas. En esta oportunidad analizo tres enfoques que compartirían esta premisa: (a) la orientación a "grandes desafíos", (b) la investigación e innovación responsable para tecnologías emergentes y (c) la innovación para la inclusión social. A continuación, analizo si puede afirmarse que existe un giro de los enfoques sobre políticas de CTI que las aleja de los objetivos económicos genéricos, al que denomino giro poscompetitivo. Finalmente concluyo que no puede hablarse de un marco alternativo coherente y sistemático pero sí de una conciencia compartida de las limitaciones de las políticas convencionales de CTI para abordar temas de interés social cuyo rédito económico no es evidente.

Palabras claves: Política de ciencia y tecnología. Innovación responsable. Grandes desafíos. Innovación inclusiva.

\footnotetext{
* Universidad Nacional de Quilmes (Argentina)
} 


\section{Is there a "post-competitive turn" in science and technology policy?}

\section{Abstract}

Science, technology and innovation (STI) policies usually refer to economic growth and competitiveness as its main objectives. In this article, I argue that in recent years conceptual frameworks in STI policies have emerged that discuss the adequacy of conventional policies to situations characterized by specific social challenges. These new frameworks give greater importance to social objectives rather than economic goals. I introduce three approaches that share this premise: (a) orientation to "grand challenges", (b) Responsible research and innovation for emerging technologies and (c) innovation for social inclusion. In turn, I analyze whether these frameworks imply a shift away from generic economic objectives (a "post-competitive" turn). Finally, I conclude that we cannot speak of a coherent and systematic alternative framework but there is nevertheless a shared awareness of the limitations of conventional STI policies to address social issues where the economic return is not evident.

Keywords: Science and Technology policy. Responsible innovation. Grand Challenges. Inclusive innovation

\section{Introducción}

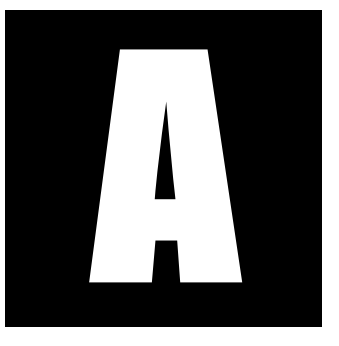

lo largo del tiempo, los marcos teóricos que guían el diseño de las políticas de ciencia, tecnología e innovación son paulatinamente renovados. En la primera etapa de la institucionalización de estas áreas de la política, luego de la segunda guerra mundial, los conceptos privilegiados fueron aquellos ligados al modelo lineal (Godin, 2006). La premisa fundamental de este enfoque se relacionaba con la necesidad de impulsar en primer término las actividades de ciencia básica, pues luego de un modo lineal y natural, éstas impulsarían las actividades de desarrollo 
tecnológico. De este ciclo surgiría finalmente el desarrollo socioeconómico. Más tarde, en la década de 1960, comenzó la discusión - por la inclusión de prioridades y criterios externos asociados a objetivos políticos o nacionales (Shils, 1969). Estas discusiones en parte fueron motivadas por el reconocimiento de la escasez de recursos y la imposibilidad de dar respuesta a todas las líneas de investigación posibles que se abren. En este sentido, si bien no necesariamente la idea de que la ciencia básica es necesaria y puede generar desarrollos beneficiosos fue abandonada, también se comenzó a tomar en cuenta que hay ciertas áreas que son más relevantes o importantes por los objetivos socio-económicos a los que pueden contribuir. Merecen entonces un financiamiento prioritario. De estos planteos surgieron más tarde marcos como el de la "investigación estratégica" (Irvine; Martin, 1984) o el cuadrante de Pasteur (Stokes, 1997), que aluden a una investigación básica orientada por demandas tecnológicas y sociales antes que por la mera curiosidad.

A partir de la década de 1980, comenzó a tomar cuerpo un marco conceptual centrado en la economía evolucionista y la idea de innovación (Elzinga; Jamison, 1996). El objetivo principal es fortalecer los llamados "sistemas de innovación". Bajo esta denominación se agrupan los diferentes actores (estatales, académicos, privados) que forman parte del proceso que da lugar a nuevas tecnologías. Se presupone que la existencia de sistemas de innovación fuertes y dinámicos contribuye a objetivos económicos de importancia, como la competitividad de la economía. La generación de innovaciones que produzcan un valor económico se vuelve el medio privilegiado a través del cual la ciencia y la tecnología traen beneficios para la población y la economía de un país, a través de la agregación de valor a los productos y la generación de empleo calificado (Lundvall, 1992; Nelson, 1993).

En este artículo propongo discutir algunos cambios recientes en el dominio de los marcos teóricos que guían las políticas de CTI. La hipó- 
tesis que planteo es que estas transformaciones forman parte de lo que denominaré un giro poscompetitivo. En la sección siguiente me ocupo de dar una definición preliminar del este concepto y plantear en qué se diferenciaría del modelo dominante en las políticas de CTI. Luego analizo tres tendencias recientes en este campo que intentan vincular CTI con temas tan diversos como grandes desafíos globales, tecnologías emergentes e inclusión social. Finalmente busco precisar hasta dónde estos desarrollos pueden encolumnarse bajo una misma corriente.

\section{El enfoque convencional y la noción del giro poscompetitivo}

La idea de que existe un giro poscompetitivo se sustenta en que ciertas afirmaciones usualmente enunciadas por los organismos internacionales como el núcleo conceptual de las políticas de CTI han sido recientemente matizadas y discutidas. Si bien las críticas proceden de marcos diversos y tienen distintos grados de radicalidad, coinciden en afirmar que el enfoque convencional no es adecuado para circunstancias específicas, en las que deben postularse marcos alternativos o complementarios. Se distancian del postulado que sostiene que los logros económicos genéricos como la competitividad o el crecimiento son el paso principal e imprescindible para que la CTI aporte al mejoramiento de las condiciones de la población. En este artículo analizo tres marcos en los que podría evidenciarse esta vocación reformista. Los dos primeros se vinculan con problemáticas compartidas por países en desarrollo y países desarrollados mientras el tercer tema es específico de las políticas de CTI para países en desarrollo. Cabe destacar que dado que los dos primeros enfoques son recientes y han sido desarrollados fundamentalmente en Europa y los Estados Unidos, éstos no son muy conocidos o discutidos en América Latina, por lo cual un objetivo secundario de este trabajo también es introducir estos debates en el contexto regional latinoamericano. 
La primera temática se vincula con la capacidad de la ciencia y la tecnología de hacer frente a los llamados "grandes desafíos" que plantean temas globales como el cambio climático o las pandemias. En este caso, la crítica pasa por el planteo de que la competencia internacional y la búsqueda de competitividad de cada economía no es el camino para responder a estas demandas y que se vuelven necesarios enfoques alternativos. En segundo lugar, el desarrollo y la difusión de tecnologías emergentes plantean desafíos que no pueden capturarse apelando meramente a factores económicos, como ha demostrado el caso de los cultivos OGM y se señala la necesidad de generar dispositivos políticos que permitan una evaluación más multidimensional de los riesgos y beneficios implicados. En tercer lugar, el vínculo lineal entre crecimiento, desarrollo e inclusión social conocido como la hipótesis del derrame - y presente en documentos de organismos como el Banco Mundial ${ }^{1}$ - es discutido por perspectivas que sostienen que las políticas convencionales de CTI no disminuyen sino que acrecientan la desigualdad. El cuadro 1 resume los puntos clave en los que los enfoques analizados se diferencian de las políticas convencionales de CTI.

\footnotetext{
${ }^{1}$ En un material educativo del Banco Mundial (2013) se afirma por ejemplo que "de acuerdo con la teoría económica, las innovaciones tecnológicas bien difundidas producirán un aumento de la productividad y las actividades económicas de un país y llevarán eventualmente a mayores salarios y a un mayor bienestar para los ciudadanos"
} 
Cuadro 1. Enfoques analizados en el trabajo como parte del giro poscompetitivo

\begin{tabular}{|l|l|l|}
\hline \multicolumn{1}{|c|}{ Postulado tradicional } & \multicolumn{1}{|c|}{ Postulado alternativo } & \multicolumn{1}{|c|}{ Nuevo enfoque } \\
\hline $\begin{array}{l}\text { La dinámica del mercado } \\
\text { es suficiente para abordar } \\
\begin{array}{l}\text { los principales tópicos de } \\
\text { interés social y político }\end{array}\end{array}$ & $\begin{array}{l}\text { Existen desafíos centrales } \\
\text { para nuestras sociedades } \\
\text { que constituyen fallas de } \\
\text { mercado }\end{array}$ & Grandes desafíos \\
\hline $\begin{array}{l}\text { La adopción o el rechazo } \\
\text { de tecnologías emergentes } \\
\text { se basa fundamentalmente } \\
\text { en criterios económicos. }\end{array}$ & $\begin{array}{l}\text { El desarrollo de tecnologías } \\
\text { emergentes involucra valo- } \\
\text { res sociales y políticos que } \\
\text { determinan su trayectoria }\end{array}$ & Innovación responsable \\
\hline $\begin{array}{l}\text { La CTI contribuye a la in- } \\
\text { clusión social a través del } \\
\text { crecimiento económico y } \\
\text { la consecuente disponibili- } \\
\text { dad de empleos mejor re- } \\
\text { munerados }\end{array}$ & $\begin{array}{l}\text { Si no se arbitran medidas } \\
\text { específicas para garantizar } \\
\text { la inclusión social, las polí- } \\
\text { ticas de pueden excluir } \\
\text { antes que incluir. }\end{array}$ & $\begin{array}{l}\text { Innovación para la el desar- } \\
\text { rollo inclusivo / Tecnologías } \\
\text { sociales }\end{array}$ \\
\hline
\end{tabular}

Fuente: Elaboración propia

En lo que sigue describiré cada uno de estos nuevos enfoques, precisando su origen y sus puntos de unión y ruptura con las políticas de CTI convencionales. Finalmente, analizaré si hay elementos para afirmar que forman parte de una misma corriente, y de ser así, señalaré sus alcances y características ${ }^{2}$.

\section{La CTI ante los "grandes desafíos"}

El concepto de "grandes desafíos", que se ha introducido en la jerga de la política científica europea a partir de la Declaración de Lund (Unión Europea, 2009), responde principalmente a la necesidad de mostrar que

${ }^{2}$ Cabe destacar que si bien estos enfoques alternativos en muchos casos ya han sido traducidos en políticas públicas, el foco de este trabajo está puesto en la dimensión conceptual que sostienen y no en las experiencias de su implementación. 
los objetivos de estas políticas están más cerca de las necesidades de la población en general y no solo de metas técnico-económicas que generan menos legitimidad para estas intervenciones. En este sentido, representan un cambio de discurso frente a las políticas clásicas de innovación implementadas desde mediados de los años ochenta, que se centraron fuertemente en el apuntalamiento de la competitividad económica a través del agregado de valor. Por otra parte, su compromiso con objetivos claros y de fácil representación por el público general las emparientan con las políticas científicas mission-oriented implementadas a partir de la segunda guerra mundial, cuyos mayores exponentes fueron los proyectos Manhattan y Apolo. A continuación describiré brevemente cuáles son las características que pueden adscribirse a este nuevo discurso, para luego plantear sus diferencias con los otros dos tipos de políticas mencionadas: las tradicionales de innovación y las ligadas a grandes proyectos como Manhattan y Apolo.

En base a un informe del EU-SPRI, foro europeo sobre políticas de innovación, pueden identificarse al menos seis características de este nuevo discurso sobre "grandes desafíos" (Kallerud et al., 2013). En primer lugar, se trata de políticas que hacen una apuesta más grande que las políticas tradicionales, en la medida en que se refieren a solucionar problemas de una envergadura mayor y con impacto de largo plazo. Esto implica también un mayor compromiso a nivel de recursos materiales e intelectuales que deberán asignarse a estos objetivos. Uno de los desafíos mencionados recurrentemente es el cambio climático y el calentamiento global, que conlleva significativas amenazas a las sociedades y ecosistemas. En estrecha vinculación con esto, una segunda característica se relaciona no sólo con una apuesta mayor sino también con un alcance mayor, en tanto los afectados por estos desafíos no estarían restringidos a un perfil de ciudadano o a un país o región determinada. Existe una tensión en torno a si se trata no sólo de grandes desafíos sino también de desafíos globales. 
Esta segunda característica parecería hablar a favor de la segunda opción, que es preferida por documentos de organismos internacionales como la OCDE (2010) que hablan directamente de desafíos globales. En tercer lugar, esta dimensión global Ilama a un enfoque colaborativo antes que competitivo entre todos los actores involucrados. Esto implica un cambio frente a las políticas de innovación tradicionales que hacen énfasis en la competitividad de las diferentes economías (subnacionales, nacionales y regionales). En este caso el enfoque busca plantear que dada la envergadura y el alcance de los problemas, es necesario instrumentar mecanismos de colaboración internacional para una situación que tiene consecuencias negativas para todos. Una cuarta característica se vincula con la naturaleza social antes que económica de los objetivos que guían estas políticas. Si bien naturalmente existe un solapamiento entre lo que podemos considerar objetivos económicos y objetivos sociales, se argumenta que en el caso del enfoque de grandes desafíos el centro de gravedad de la política se encuentra antes en lo social que en lo económico. Esto no implica que se abandone la aspiración al crecimiento económico y la competitividad o que se excluya al sector privado. En todo caso, ambas esferas deben combinarse en función de lograr objetivos más amplios. Las empresas pueden ver estos desafíos como una oportunidad de negocios. Se trataría de juegos en los que todos ganan, pues habría tanto un potencial de mercado como una necesidad social de resolver un problema importante. En quinto lugar, se trata de políticas que buscan claramente orientar la investigación y ser muy directivas al respecto. Aquí sí habría un reconocimiento de una situación de falla de mercado que explicaría que este tipo de políticas son necesarias, pues no puede confiarse en que la dinámica normal del mercado va naturalmente a abocarse a estas cuestiones. Por último, cabe señalar que se trata fundamentalmente de políticas orientadas a la innovación y no tanto a la producción de conocimientos 
básicos o de propósito general. Se espera que este tipo de políticas produzcan resultados concretos en el mundo real, que contribuyan a la solución de los desafíos que se planteaban.

La similitud de este discurso de los "grandes desafíos" con las políticas tecnológicas asociadas a la Big Science no es difícil de reconocer. En ambos casos se trata de un desafío concreto a resolver y para el cual se movilizan una cantidad extraordinaria de recursos de la más alta calidad. Así es que algunos, como Talbot (2006) se permiten las analogías y plantean la necesidad de un "programa Apolo" para el sector energético. Sin embargo, autores como Mowery, Nelson, y Martin (2010), Ilaman la atención sobre las diferencias radicales entre estos nuevos "grandes desafíos" y los viejos desafíos representados por el proyecto Apolo y Manhattan. Por ello, señalan que para el caso del cambio climático -y esto sería extensible a otros desafíos usualmente mencionados como la cura del cáncer, la seguridad alimentaria, el envejecimiento poblacional o el terrorismo- no se necesita "vino nuevo en odres viejos" sino un diseño de política radicalmente innovador.

La inadecuación de las políticas mission-oriented de la Big Science para estos nuevos desafíos se basa en que la naturaleza y el contexto de los problemas planteados es radicalmente distinta. A diferencia del caso Manhattan o Apolo, donde lo que se buscaba era un producto específico, aquí deben desarrollarse una variedad de productos y procesos, que requieren no sólo de innovación tecnológica sino también de innovación social y cambios culturales. El cambio climático o los problemas planteados por una sociedad que envejece no serán solucionados con una bala de plata tecnológica, a la manera que el estallido de Fat Man y Little Boy dieron por cumplidos los objetivos del proyecto Manhattan. Por otra parte, en los nuevos desafíos no hay un único cliente -que es además el financiador del proyecto- como lo era el gobierno federal norteamericano en los casos mencionados. Ahora se trata de una diversidad de actores, 
cada uno con intereses muchas veces contrapuestos, que requieren un enfoque mucho más descentralizado. Estos actores, por otra parte, no necesariamente han asumido un compromiso con la adopción de la nueva tecnología, y es por eso que trabajar para facilitar la adopción debe ser un aspecto importante de las políticas, que puede lograrse a través de regímenes de propiedad intelectual no tan restrictivos, que tengan amplias facilidades para el licenciamiento (Foray et al., 2012).

El discurso de los grandes desafíos retiene de la política tradicional de innovación la importancia de involucrar al sector privado y de integrar lo público y lo privado. Al contrario, se fomentan posibles alianzas en el marco de un enfoque sistémico de la innovación. Sin embargo, los objetivos principales explícitos que se persiguen no están necesariamente en la misma línea de las políticas tradicionales de innovación, orientadas a aumentar el crecimiento económico, la competitividad y la agregación de valor. En este caso, en cambio, los sistemas de innovación deberán orientarse a objetivos de corte más social y con un perfil menos competitivo. Esto no implica abandonar la dimensión económica, pero sí subordinarla a ciertas metas que tienen su fundamento en desafíos compartidos por toda la sociedad, que sean más visibles y específicos. En la medida en que se trata de apuestas que en una primera fase pueden ser muy riesgosas, aparecen en escena mecanismos propios de situaciones de falla de mercado, como las licitaciones y la compra pública o los premios de innovación. Tampoco la política de propiedad intelectual que autores como Mowery, Nelson y Martin (2010) proponen es la clásica de las políticas de innovación, en la medida en que en estos casos el "efecto demostración" es muy importante y por lo tanto debe adoptase un enfoque que favorezca la diseminación, a través de la libre disponibilidad de licencias con royalties razonables.

En base a lo mencionado recientemente, puede notarse que el discurso sobre los grandes desafíos involucra algunas novedades, y por lo 
tanto, creo que puede considerarse que contiene componentes novedosos. Pero se trata más bien de una suerte de innovación incremental antes que radical, en la medida en que combina elementos preexistentes sin sustituirlos ni discutir su fundamento. Su novedad consiste en apartar el foco central en la competitividad y el crecimiento económicos y crear una representación de los objetivos de estas políticas más cercana al ciudadano de a pie. Por último, puede señalarse que el contenido de los desafíos que aparecen citados en la bibliografía posee un cierto sesgo hacia problemas de mayor importancia para los países desarrollados o para ciertos sectores de su economía. Las cuestiones ligadas a la desigualdad social, desempleo, educación y pobreza, por ejemplo, no aparecen en primer plano (Kaldewey, 2013).

\section{Innovación responsable: nuevas formas de integrar al público}

Paralelamente a la discusión mencionada en el apartado anterior, en los últimos años emergió también el concepto de "Investigación e Innovación Responsable" (RRI, por sus siglas en inglés), que ha ganado particular importancia en las políticas de CTI de la Unión Europea (Owen et al., 2013). Si la preocupación por la orientación de la política científica a los "grandes desafíos" se centra fundamentalmente en modificar las prioridades de investigación y acercarlas a problemas más palpables y concretos que afectan directamente a mayor número de personas, lo que propone la innovación responsable no es solo discutir el qué investigar sino fundamentalmente el cómo. En el caso de la innovación responsable, el centro de la cuestión está en garantizar un modo particular de proceder, vinculado con la generación de mecanismos participativos que se pongan en práctica durante el transcurso de las investigaciones y moldeen su desarrollo. Es decir, en el enfoque 
de grandes desafíos la participación de los actores y potenciales afectados parece restringida -o al menos solo impulsada- en las etapas previas de discusión en torno a qué grandes desafíos deben ser abordados. Luego, son los políticos, científicos y empresarios que determinan la mejor forma de llevar a cabo estos objetivos. En el caso de la innovación responsable, la participación de todos los potenciales involucrados se da durante todo el proceso, e incluye explícitamente a legos y usuarios.

El enfoque de la RRI está ligado directamente con la pregunta por la gobernanza de las tecnologías emergentes (Guston, 2014). Se origina en el contexto de las investigaciones sobre nanotecnología, fundamentalmente en el marco de la National Nanotechnology Initiative en los Estados Unidos a comienzos de la década de 2000, bajo la idea del "desarrollo responsable" (Roco et al., 2011). Este enfoque es heredero de las investigaciones previas en torno a potenciales consecuencias indeseadas del desarrollo científico y tecnológico. Estas cuestiones, que en un comienzo fueron abordadas por la filosofía de la ciencia, la bioética o la evaluación tecnológica, tuvieron un nuevo impulso a comienzos de la década de 1990 con el componente ELSI (Ethical, Legal and Social Implications) del Proyecto Genoma Humano. Luego, en 1994 en el contexto europeo, se propuso cambiar el acrónimo ELSI por ELSA sustituyendo la idea de "implicaciones" por la de "aspectos" éticos, legales y sociales. De esta manera, se intentaba salir de una visión lineal respecto de los impactos. Una de las cuestiones importantes era plantear los aspectos con implicancias potencialmente más difusos y que requieren un mayor grado de participación pública, y no quedar restringido a temas que podían fácilmente ser encuadrados y trabajados únicamente con conocimiento experto, como los de riesgo, seguridad y salud (Thoreau, 2013; Zwart et al., 2014).

A poco tiempo de estar consolidándose la investigación en el campo de ELSI/ELSA, que tuvo su auge en la primera década de los 2000, 
surge entonces - sobre la base de la investigación en nanotecnología- el marco de la Investigación e Innovación Responsable (RRI). Éste se planteó como una suerte de concepto superador, que pretendía englobar el trabajo sobre ELSA pero darle una dimensión más amplia e integrarlo con otros espacios más amplios de la política científica. Para comprender la genealogía de este enfoque, conviene retrotraerse al debate sobre las biotecnologías y particularmente los organismos genéticamente modificados (OGM). En ese contexto se generó en Europa una visión pública potencialmente crítica ante las tecnologías emergentes, al punto tal que Rip (2006) señaló que existía una "nanofobia-fobia", es decir, un gran miedo dentro de la comunidad científica e industrial a que se repita para el caso de la nanotecnología el rechazo de grandes sectores del público europeo que sufrieron las biotecnologías. El concepto de RRI amplió luego su alcance más allá del campo específico de la nanotecnología y hoy ocupa un lugar central en el programa de financiamiento de la CTI Horizon 2020, en el marco del cual constituye uno de los conceptos rectores del componente "Science with and for Society", cuyo presupuesto total para 2014 y 2015 es de 91 millones de euros (Unión Europea, 2014; Galiay 2014).

No puede decirse que haya una única definición de RRI ${ }^{3}$, sino elementos que se asocian agregativamente a la misma. De acuerdo con Zwart et al. (2014), esto podría dar cuenta de que se planteó desde arriba el significante con anterioridad a la fijación del significado. Sin embargo, sí es posible distinguir algunas notas características que aparecen en la mayoría de los autores que utilizan la expresión "RRI", que habilita a pensar que existe un marco más o menos definido. Stilgoe et al (2013) señalan que, a su juicio, lo que caracteriza a la innovación responsable es la intención de generar espacios de deliberación sobre aspectos de las in-

\footnotetext{
${ }^{3}$ Lo más cercano a un marco conceptual oficial de la UE es el que se desprende de los textos de von Schomberg (2014), pero no deben tomarse como una visión de consenso.
} 
novaciones que generan preocupación o interés público. De esta manera, con la participación de todos los actores afectados (gobierno, academia, industria, sociedad civil), se espera que las innovaciones producidas reflejen más adecuadamente los valores e intereses de un conjunto más amplio de actores y no sólo los de los promotores de la tecnología.

También se han sumado voces críticas con el enfoque de RRI en la medida en que ven en él un sesgo pro-industria, que, al institucionalizar y promover en forma top-down la participación ciudadana limitarían el potencial crítico que habrían permitido previamente los mecanismos participativos ${ }^{4}$ que se difundieron a partir de la década de 1990 (Thoreau, 2013). En el caso de la RRI, lo que se buscaría con la participación de todos los actores "corriente arriba" principalmente es evitar el surgimiento de una oposición y un movimiento resistencia radical a las tecnologías (Bauer, 1995) y no necesariamente provocar una apertura democrática. En este sentido, se argumenta que la RRI sería aliada del desarrollo tecnológico tradicional, permitiendo "detenerse a pensar un minuto", pero sin poner verdaderamente en tela de juicio el avance tecnológico y sus beneficios generales (RIP, 2014). Por último, se ha señalado también un riesgo a la burocratización de las evaluaciones de RRI, que pueden transformarse en puntos de pasaje obligados pero no necesariamente reflexivos para los actores involucrados.

El marco de RRI entonces parte de una visión mucho más amigable con el mercado y la innovación que los planteos más radicales previos, ligados a la participación pública en la evaluación de tecnologías. En este sentido, la innovación responsable es una propuesta mucho más reformista que revolucionaria. Su diferencia fundamental frente a otros enfoques más convencionales es que sostiene que la difusión y apropiación social de tecnologías emergentes no es un fenómeno a analizar en términos

\footnotetext{
${ }^{4}$ Entre ellos podemos contar al Technology Assessment, las conferencias de consenso, los jurados ciudadanos, etc.
} 
exclusivamente económicos (como hacen trabajos como el de Bozeman et al, 2008). El marco de RRI supone tanto un elemento descriptivo ("la adopción de una tecnología debe comprenderse desde una perspectiva multidimensional") como uno normativo ("Debe facilitarse la participación del mayor número de actores en etapas tempranas del desarrollo") que abogan en contra del reduccionismo económico y del monopolio del conocimiento experto.

\section{Innovación, desigualdad e inclusión social}

La aparición de los nuevos conceptos de "innovación responsable" y "grandes desafíos" en el campo de la política científica se dio fundamentalmente en los Estados Unidos y la Unión Europea, de donde proceden los análisis y referencias desarrollados en las secciones previas. Esto no significa sin embargo que otras regiones del mundo hayan permanecido ajenas a estas transformaciones. En el último tiempo, asistimos a un creciente interés por vincular la política de ciencia, tecnología e innovación de modo más directo con la problemática de la desigualdad y la inclusión social. Esta tendencia se verifica tanto de parte de los organismos internacionales como el Banco Mundial o la OCDE (Paunov, 2013; Dahlman et al., 2014) como de investigadores académicos (Cozzens; Sutz, 2012; Casas et al., 2014). Si bien en ambos casos, se reconoce la necesidad de políticas de CTI específicas para abordar los desafíos de la desigualdad y la pobreza, los enfoques adoptados por los organismos internacionales se centran en la necesidad de incluir a la "base de la pirámide" como consumidores, y ven a las innovaciones inclusivas como un medio de impulsar la senda del crecimiento económico y la competitividad ampliando mercados (ver por ejemplo Prahalad; Mashelkar, 2010)5. En contraposi-

${ }^{5}$ Para una comparación más detallada de estos enfoques, véase Gras y Bortagaray (2014) y Thomas, Bortz y Garrido (2015) 
ción, las visiones académicas mencionadas plantean a los beneficiarios no solamente como consumidores sino como ciudadanos y como innovadores. Son fundamentalmente entonces estas últimas perspectivas las que podrían enmarcarse dentro del giro poscompetitivo discutido aquí. A continuación describiré las dos concepciones principales en América Latina sobre la cuestión: las Tecnologías Sociales y el marco de Innovación para la Inclusión Social.

\subsection{Tecnologías Sociales}

Uno de los teóricos latinoamericanos más activos en torno a la vinculación entre tecnología y procesos de inclusión y exclusión social es Hernán Thomas. Junto con su equipo en el Instituto de Estudios sobre la Ciencia y la Tecnología de la Universidad Nacional de Quilmes han desarrollado un marco teórico que se propone dar fundamento a instrumentos de política de CTI orientados a fomentar la inclusión social y el desarrollo sostenible (Thomas et al., 2012; Thomas, 2012). El planteo de estos autores se basa en una lectura crítica de los modelos previos de vinculación entre tecnología y pobreza, fundamentalmente los ligados a las "tecnologías apropiadas". Si aquellos planteaban un modelo de desarrollo tecnológico jerárquico y paternalista, que buscaba acercar ciertas tecnologías a un costo menor y con características adecuadas a las necesidades de los potenciales usuarios, la propuesta del grupo de Thomas busca hacer foco no sólo en el producto tecnológico y su apropiación o adecuación funcional a las necesidades e intereses de la población objetivo, sino detenerse también en las características del proceso de diseño de los nuevos sistemas socio-técnicos.

Las tecnologías para la inclusión social o tecnologías sociales, núcleo de la propuesta teórica de este grupo, se basan en un proceso participativo democrático de co-construcción de nuevos sistemas socio-técnicos 
entre los distintos actores involucrados, que pueden incluir académicos y expertos, pero fundamentalmente se encuentra guiado en una forma bottom-up por las necesidades y modos de organización social de aquellos cuyo problema la tecnología busca abordar. Es por ello que los actores privilegiados para la construcción de este tipo de tecnologías son los movimientos sociales y las cooperativas y no las universidades o las fundaciones filantrópicas. Cabe destacar que el énfasis en las tecnologías organizacionales acerca también a este marco a las discusiones sobre innovación social, con la particularidad de que el factor tecnológico ocupa en el marco de tecnologías sociales un factor mucho más crucial y predominante que en las discusiones sobre innovación social (Howaldt; Schwarz, 2010). Finalmente, es importante señalar que se busca que las intervenciones generadas tengan un efecto inclusivo, es decir, que potencien la construcción de ciudadanía de las comunidades receptoras y no tengan un efecto estigmatizante, en el sentido de que los artefactos producidos sean fácilmente identificados como productos para pobres.

Por otra parte, el planteo de estos autores se enmarca en una fuerte crítica no sólo de los movimientos de tecnologías apropiadas, sino también de las políticas imperantes en materia de ciencia, tecnología e innovación. El marco se alimenta de una crítica a todo el paradigma de la economía evolucionista y los sistemas nacionales de innovación (Dagnino; Thomas, 2009). La crítica a estas teorías se basa en la aplicación mecánica a las economías periféricas de los modelos de política pensados para países desarrollados (Delvenne; Thoreau, 2012). Dada la falta de demanda interna de conocimientos, las comunidades académicas dominan el policy-making en CTI e impulsan estrategias para beneficio interno de sus intereses disciplinares. Si hay una vinculación con actores externos que se ve potenciada, es con el sector privado empresario, al cual se transfieren recursos que no redundan en un beneficio para toda la sociedad (Dagni- 
no, 2007). Siguiendo a autores como Feenberg, sostienen que el privilegio del interés privado por sobre el público en el desarrollo tecnológico lleva a que la ciencia y la tecnología incluyan en su estructura interna reglas de juego y valores intrínsecos al sistema capitalista (Dagnino, 2008). Las políticas públicas que impulsen el desarrollo de tecnologías sociales entonces deberán estar orientadas en un sentido nuevo. No deberán seguir el patrón clásico del modelo lineal de políticas de empuje de la ciencia ni tampoco encolumnarse detrás de la tracción de la demanda, en el sentido schumpeteriano. Se trata de hacer énfasis en las interacciones y construir capacidades estratégicas para dar soluciones no-lineales que empoderen a los actores menos privilegiados.

\subsection{Innovación para el desarrollo inclusivo}

Además del marco de las "tecnologías sociales" mencionado, ha ganado también preminencia en la región el marco de la "innovación para la inclusión social" (Arocena; Sutz, 2009). Este enfoque, impulsado fundamentalmente por el grupo de Judith Sutz en la Universidad de la República de Uruguay, comparte con el marco previo la importancia de vincular las capacidades de ciencia, tecnología e innovación con el desafío de la inclusión social. A su vez, comparten la crítica a la orientación predominante de las políticas de $\mathrm{CTI}$, que no han demostrado ser eficaces en abordar el tema de la inclusión social a través del crecimiento económico y el aumento de la competitividad. Este enfoque sin embargo marca algunas diferencias importantes frente al de las tecnologías sociales.

La principal diferencia radica en que el enfoque de Sutz y su equipo está pensado para acciones realizadas desde la universidad. Es decir, partiendo de una crítica a los modos predominantes de producción de conocimiento académico, perfilan una nueva opción, diferente tanto a la de producir conocimiento disciplinar esotérico como a la orientar la I+D 
a las necesidades productivas para fomentar la innovación en el sector privado. Esta nueva opción, ligada a producir innovaciones orientadas a la inclusión social, se nutre del conocimiento académico, y propone asociarlo a intereses que no estaban necesariamente representados previamente en la política de CTI sino confinados a las políticas sociales. En los ejemplos los autores, destacan el papel del Estado como demandante de innovaciones de bajo costo que permitan hacer frente a situaciones donde la tecnología se transforma en un factor de exclusión social (Alzugaray et al., 2011). El diseño de focos LED de más bajo costo para tratar la ictericia en bebés prematuros o de una piel sintética desarrollada localmente son situaciones en las cuales se ha logrado desarrollar a menor costo un producto que ya existía en el mercado, pero no era accesible para importantes grupos de la población de un país en desarrollo.

Al tratarse fundamentalmente de un enfoque que busca poner el conocimiento académico a disposición de las necesidades de actores marginados, se parte del supuesto de que se trata de que el conocimiento experto es potencialmente útil y valioso, y esto configura un modelo de interacción mucho más top-down que en el marco de las tecnologías sociales, donde el actor privilegiado que motoriza el desarrollo no es la comunidad universitaria -en alianza con las políticas públicas- sino los movimientos sociales de base. La participación de grupos de investigación universitaria provoca además que los procesos analizados tengan un piso de complejidad tecnológica más alta que los que pueden surgir de los movimientos sociales, los cuales pueden ser igualmente complejos, pero también ser low-tech ${ }^{6}$.

Por otra parte, si bien hay una crítica a las políticas de innovación clásicas y a su orientación a la competitividad, no se plantea una ruptura

\footnotetext{
${ }^{6} \mathrm{Si}$ bien la mayoría de los casos presentados por quienes proponen las tecnologías sociales son de baja tecnología autores como Fonseca y Pereira (2014) analizan la posibilidad de aplicar el marco de tecnologías sociales a tecnologías emergentes como la nanotecnología.
} 
radical con la economía evolucionista y el modelo de Sistemas de Innovación, como en el marco de las tecnologías sociales, que en algunos textos prefieren no hablar siquiera de "innovación". Tampoco hay, como en el marco de las tecnologías sociales un intento por hacer una crítica epistemológica a la tecnología convencional y establecer una vinculación intrínseca con el capitalismo. En el planteo uruguayo, no se trata necesariamente de crear nuevos paradigmas tecnológicos, sino adaptar los existentes a contextos de escasez y desigualdad.

En última instancia, si bien ambos marcos acentúan la cuestión del desarrollo, es claro que las concepciones de desarrollo que hay detrás de cada iniciativa son divergentes. El marco de Sutz et al. no reniega de objetivos de desarrollo universales como podrían ser los marcados por la agenda de Naciones Unidas. En la línea de la discusión sobre desarrollo y dependencia de la década de 1960, hace hincapié en la importancia de pensar el subdesarrollo como un fenómeno específico y no sólo como retraso y necesidad de modernización (Alzugaray et al., 2013).

El marco de las tecnologías sociales en cambio tiene una postura más radical y se acerca a la agenda post-desarrollista (Rist, 2002). De acuerdo con esta perspectiva, no pueden pensarse objetivos de desarrollo universales. No hay una única forma deseable de desarrollo ni de "buena vida". Cada cultura tiene sus formas propias y específicas de considerar lo que significa el desarrollo. Por tanto, las metas universales pueden encubrir intentos de homogeneización o imperialismo cultural que deben ser evitados. La inclusión de nuevas tecnologías no es de por sí buena y deseable si no están adecuadas a las particularidades culturales de la sociedad en la que se insertarán. A nivel económico, el post-desarrollismo rompe lanzas definitivamente con los marcos basados en el crecimiento y la competitividad y aboga por visiones más radicales como las vinculadas al decrecentismo (Jackson, 2011). 


\section{6. ¿Estamos ante un giro poscompetitivo en la política de CTI?}

Al comenzar el trabajo, propuse la hipótesis de que podría estar dándose una transformación en los marcos que guían la política científica, que marcaría una renovación frente al consenso de las políticas de innovación orientadas al crecimiento y la competitividad. Analicé entonces tres marcos teóricos recientes para las políticas de CTI que serían parte de esta renovación: el ligado a grandes desafíos, la investigación e innovación responsable y la innovación para la inclusión social. Cabe preguntarse ahora si existen rasgos comunes que permitan agrupar a estos desarrollos dentro de una única tendencia, que denominé el giro poscompetitivo. La idea principal de este giro sería que existe una nueva generación de conceptos que guían las políticas de CTI que desplazan al crecimiento y la competitividad económica como objetivos centrales a los que la ciencia y la tecnología debería aportar. En principio, no se niega que éstos puedan ser importantes o relevantes, pero se quita el foco central en ellos como razón suficiente para la legitimar la inversión pública en CTI.

Una primera apreciación conjunta de los tres marcos estudiados nos permite concluir que no todos son incompatibles con las políticas tradicionales de innovación. El marco de "grandes desafíos", por caso, puede plantearse como una aproximación complementaria a las políticas convencionales para temas que requieren inversiones más grandes, y colaboración internacional antes que competencia. En este sentido, no descree de la competitividad y el crecimiento como una un objetivo deseable. De hecho, se plantea que los grandes desafíos pueden constituir oportunidades de negocio ${ }^{7}$. El punto a destacar es el reconocimiento de que existen ciertos temas en los que un abordaje convencional no es el

\footnotetext{
${ }^{7}$ Es conveniente destacar que autores como Richard Nelson que hoy proponen estos enfoques frente a grandes desafíos estuvieron entre los principales impulsores de las políticas basadas en los Sistemas de Innovación en la década de
} 
más adecuado. La innovación responsable tampoco está completamente reñida con los marcos tradicionales. De hecho, he señalado cómo para algunos analistas, la innovación responsable supone una forma de abordar la potencial conflictividad ligada a las tecnologías emergentes de un modo más pro-mercado y que facilita antes que bloquea la innovación. Es decir, plantea que es necesario un acompañamiento más multidimensional al desarrollo de tecnologías emergente. No reniega de la inclusión de criterios económicos, lo que critica es una postura reduccionista al respecto. En tercer lugar, los marcos vinculados a innovación e inclusión social, no son unívocos respecto a su interacción con las políticas tradicionales de CTI. Si bien los dos marcos descritos son críticos frente a la capacidad de la las políticas de innovación de contribuir a la inclusión social, sólo el de tecnologías sociales plantea una crítica completa a la economía de la innovación. De acuerdo a estos últimos, la tecnología que se crea en una sociedad capitalista incorpora valores ligados a ese sistema económico y entonces las tecnologías sociales deben crear una tecnología alternativa alejada de la lógica de mercado. En base a lo anterior, difícilmente entonces pueda pensarse a los marcos analizados y al giro poscompetitivo como una tendencia contrahegemónica en sentido fuerte, o como una apuesta a un cambio de sistema socio-económico que se distancie de la economía de mercado. Sin embargo, sí pueden señalar una tendencia reformista que complemente el marco convencional de las políticas de $\mathrm{CTI}$ con enfoques específicos que privilegian objetivos sociales antes que metas económicas genéricas.

He señalado que el giro poscompetitivo no implica necesariamente una postura de ruptura completa con la política tradicional de CTI para una agenda contrahegemónica. No es una tendencia homogénea ni coherente. Pero lo que sí recata el giro poscompetitivo es la emergencia de discursos sobre las políticas de CTI que critican el consenso existente 
sobre la economía del conocimiento y el papel de la ciencia y la tecnología en el mismo. Tanto para afrontar los "grandes desafíos" como para desarrollar tecnologías emergentes socialmente aceptadas y para que la CTI contribuya a la inclusión social, las políticas tradicionales de innovación no serían suficientes. El giro poscompetitivo entonces a lo que refiere es a la emergencia de enfoques y conceptos - que se traducen luego en instrumentos- que abordan temas específicos dentro de la gobernanza de la ciencia y la tecnología ante los cuales las políticas convencionales no logran dar una respuesta satisfactoria. Estos nuevos marcos comparten además su carácter específico, no son marcos para cualquier tipo de innovación, sino que acercan los objetivos de la CTI a imágenes que son más representativas para el público en general, como un problema específico (la inclusión, los riesgos de una nueva tecnología o un gran desafío como el cambio climático). Esto acerca estas políticas a objetivos sociales antes que a metas económicas que son más cercanas para la población en general, lo cual podría aumentar la legitimidad social de las políticas de CTI.

En conclusión, el giro poscompetitivo no implica una revolución en la política de CTI ni un abandono de las ideas de los sistemas de innovación y la economía del conocimiento. Lo que sí enfatiza es que hay ciertos aspectos que no pueden ser abordados con las soluciones tradicionales y exigen una mirada específica en las que la competitividad y el crecimiento no deben pensarse como los objetivos principales. Si bien el nombre de "giro poscompetitivo" puede sonar un poco grandilocuente para describir una tendencia reformista antes que revolucionaria, prefiero mantenerlo para resaltar que desde distintos sectores -desconectados entre sí- están surgiendo enfoques que comparten la necesidad de buscar formas novedosas de abordar desafíos que las políticas de CTI no han podido atender satisfactoriamente con su modelo estándar. 
Sociologias, Porto Alegre, ano 18, no 41, jan/abr 2016, p. 242-268

Federico Vasen es investigador postdoctoral CONICET en el Instituto de Estudios sobre la Ciencia y la Tecnología de la Universidad Nacional de Quilmes (UNQ), Argentina. Es Doctor en Ciencias Sociales y Humanas de la UNQ y graduado en Filosofía por la Universidad de Buenos Aires. Se especializa en estudios sobre políticas científicas y educación superior. $\measuredangle$ fvasen@unq.edu.ar

\section{Referencias}

1. ALZUGARAY, S.; MEDEROS, L.; SUTZ, J. Investigación e innovación para la inclusión social: la trama de la teoría y de la política. Isegoría, 48, 2013. pp. 25-50.

2. ALZUGARAY, S.; MEDEROS, L.; SUTZ, J. La investigación científica contribuyendo a la inclusión social. Revista Iberoamericana de Ciencia, Tecnología y Sociedad, 6 (17), 2011. pp. 11-30.

3. AROCENA, R; SUTZ, J. Sistemas de innovación e inclusión social. Pensamiento Iberoamericano, 5, 2009. pp. 99-120.

4. BANCO MUNDIAL. Overview of Innovation and Innovation Policy. Material del curso online Introduction to Innovation Policy in Developing Countries, disponible en http://worldbank.mrooms.net/file.php/745/Html/pdf/Presentation_ m01.pdf, 2013.

5. BAUER, M. Resistance to new technology. Nuclear power, information technology and biotechnology. Cambridge: CUP, 1995.

6. BOZEMAN, B.; HARDIN, J. LINK, A.N. Barriers to the diffusion of nanotechnology. Economics of Innovation and New Technology, 17 (7-8), 749-761, 2008.

7. CASAS, R.; CORONA, J. M.; RIVERA, R.. Políticas de Ciencia, Tecnología e Innovación en América Latina: entre la competitividad y la inclusión social. In: KREIMER, P. et al. (Eds.), Perspectivas Latinoamericanas en el Estudios Social de la Ciencia, la Tecnología y el Conocimiento. México: Siglo XXI, 2014.

8. COZZENS, S.; SUTZ, J. Innovation in informal settings: a research agenda. Trabajo para discusión en el GRIID Network, International Development Research Centre, Ottawa, 2012.

9. DAGNINO, R. Ciência e Tecnologia no Brasil: o Processo Decisório e a Comunidade de Pesquisa. Campinas: Unicamp, 2007.

10. DAGNINO, R. Neutralidade da ciência e determinismo tecnológico. Um debate sobre a tecnociência. Campinas: Unicamp, 2008. 
11. DAGNINO, R.; THOMAS, H. Planejamento e políticas públicas de inovação: em direção a um marco de referência latino-americano. Planejamento e Políticas Públicas, 23, 2009.

12. DAHLMAN, C. et al. Inclusive Innovation. Harnessing Creativity to Enhance the Economic Opportunities and Welfare of the Poor. Washington: World Bank Group / Trade and Competitiveness, 2014.

13. DELVENNE, P.; THOREAU, F. Beyond the "Charmed Circle" of OECD: New Directions for Studies of National Innovation Systems. Minerva, 50, 205-219, 2012.

14. ELZINGA, A.; JAMISON, A. El cambio de las agendas políticas en ciencia y tecnología, Zona Abierta, 75, 76, 1996.

15. FONSECA, P.F.C.; PEREIRA, T.S. The governance of nanotechnology in the Brazilian context: Entangling approaches. Technology in Society, 37, 2014. pp. 16-27.

16. FORAY, D.; MOWERY, D.C.; NELSON, R.R. Public R\&D and social challenges: What lessons from mission R\&D programs? Research Policy, 41(10), 2012. pp. 1697-1702.

17. GALIAY, P. Responsible Research and Innovation: A Cross Cutting Issue in Horizon 2020. Disponible: http://ec.europa.eu/information_society/newsroom/ cf/dae/document.cfm?doc_id=4146, 2014.

18. GODIN, B. The Linear Model of Innovation: The Historical Construction of an Analytical Framework. Science, Technology and Human Values 31, 2006. pp. 639-667.

19. GRAS, N.; BORTAGARAY, I. Science, Technology, and Innovation Policies for Inclusive Development: Shifting Trends in South America. In: CRESPI, G.; DUTRÉNIT, G. (Eds) Science, Technology and Innovation Policies for Development. The Latin American Experience. Cham: Springer, 2014. pp. 255-285.

20. GUSTON, D. Understanding 'anticipatory governance'. Social Studies of Science. 44 (2), 218-242, 2014.

21. HOWALDT, J.; SCHWARZ, M. Social Innovation: Concepts, research fields and international trends. Dortmund: Sozialforschungsstelle SFS, 2010.

22. IRVINE, J.; MARTIN, B.R., Foresight in Science: Picking the Winners. Londres: Pinter, 1984.

23. JACKSON, T. Prosperity without Growth. Londres: Earthscan, 2011.

24. KALDEWEY, D. Tackling the Grand Challenges. Reflections on the Responsive Structure of Science. Presentado en Early Career Researcher Conference (ECRC) 
Science dynamics and research systems: The role of research for meeting societal challenges, Madrid, 2013.

25. KALLERUD, E. et al. Dimensions of Research and Innovation Policies to Address Grand and Global Challenges. Oslo: Nordisk institutt for studier av innovasjon, forskning og utdanning, 2013.

26. LUNDVALL, B.A. National systems of innovation. Londres: Pinter Publishers, 1992.

27. MOWERY, D.C.; NELSON, R.R.; MARTIN, B.R. Technology policy and global warming: Why new policy models are needed (or why putting new wine in old bottles won't work). Research Policy, 39(8), 2010. pp. 1011-1023.

28. NELSON, R.R. (Ed.). National innovation systems: A comparative study. Oxford: Oxford University Press, 1993.

29. OWEN, R.; BESSANT, J.R.; HEINTZ, M. (Eds.) Responsible innovation: managing the responsible emergence of science and innovation in society. Chichester: Wiley, 2013.

30. PAUNOV, C. Innovation and Inclusive Development: A Discussion of the Main Policy Issues. OECD Science,Technology and Industry Working Papers, 2013/01, OECD Publishing, 2013.

31. PRAHALAD, C.K.; MASHELKAR, R.A. Innovation's Holy Grail. Harvard Business Review, julio-agosto, 2010.

32. RIP, A. Folk Theories of Nanotechnology. Science as Culture, 15 (4), 2006. pp. 349-365.

33. RIST, G. El desarrollo: historia de una creencia occidental. Madrid: Libros de la Catarata, 2002.

34. ROCO, M.C. et al. Innovative and responsible governance of nanotechnology for societal development. Journal of Nanoparticle Research, 13(9), 2011. pp. 3557-3590.

35. SHILS, E. (Ed.), Criteria for Scientific Development: Public Policy and National Goals. Cambridge: MIT Press, 1969.

36. STILGOE, J., OWEN, R.; MACNAGHTEN, P. Developing a framework for responsible innovation. Research Policy, 42(9), 2013. pp. 1568-1580.

37. STOKES, D. E., Pasteur's Quadrant: Basic Science and Technological Innovation. Washington: Brookings Institution Press, 1997.

38. TALBOT, D. Needed: an «Apollo Program» for Energy. MIT Technology Review News, a partir de http://www.technologyreview.com/news/405681/neededan-apollo-program-for-energy/, 20 abril 2006. 
39. THOMAS, H. Tecnologías para la inclusión social en América Latina: de las tecnologías apropiadas a los sistemas tecnológicos sociales. Problemas conceptuales y soluciones estratégicas. In: THOMAS, H.; SANTOS, G.; FRESSOLI, M. (Eds.), Tecnología, Desarrollo y Democracia. Buenos Aires: MINCyT-UNQ, 2012.

40. THOMAS, H.; FRESSOLI, M.; BECERRA, L. Science and technology policy and social ex/inclusion: Analyzing opportunities and constraints in Brazil and Argentina. Science and Public Policy, 39(5), 2012. pp. 579-591.

41. THOMAS, H.; BORTZ, G.; GARRIDO, S. Enfoques y estrategias de desarrollo tecnológico, innovación y políticas públicas para el desarrollo inclusivo. Documentos de Trabajo IESCT-UNQ, número 1, 2015.

42. THOREAU, F. Embarquement immédiat pour les nanotechnologies responsables. Comment poser et re-poser la question de la réflexivité? (Tesis de doctorado en Ciencias Políticas y Sociales). Universidad de Lieja, Bélgica, 2013.

43. UNIÓN EUROPEA. Horizon 2020 Work Programme 2014 - 2015 16. Science with and for Society. Recuperado desde http://ec.europa.eu/research/participants/data/ref/h2020/wp/2014_2015/main/h2020-wp1415-swfs_en.pdf, 2014.

44. UNIÓN EUROPEA. The Lund Declaration. Recuperado a partir de http:// era.gv.at/object/document/130, 2009.

45. VON SCHOMBERG, R. A Vision of Responsible Research and Innovation. In: OWEN, R; BESSANT, J; HEINTZ, M. Responsible Innovation. Managing the responsible emergence of science and innovation in society Londres: Wiley, 2013. pp. 51-74.

46. ZWART, H.; LANDEWEERD, L.; ROOIJ, A. Adapt or perish? Assessing the recent shift in the European research funding arena from 'ELSA' to 'RRI'. Life Sciences Society and Policy, 10(1), 11, 2014.

Recebido em: 11/03/2015

Aceite final: 10/08/2015 